\title{
CoVID-19 Respiratory specialists working in different ways: Development of a GP hotline and respiratory support service during the COVID-19 pandemic
}

\author{
Authors: Kartik Kumar, ${ }^{\mathrm{A}}$ Vincent Mak, ${ }^{\mathrm{B}}$ Katherine Groom, ${ }^{\mathrm{C}}$ Yasmin Razak, ${ }^{\mathrm{D}}$ Joanna L Brown, ${ }^{\mathrm{E}}$ Toby Hyde, ${ }^{\mathrm{F}}$ \\ Anna Bokobza, ${ }^{\mathrm{G}}$ Robina K Coker, ${ }^{\mathrm{H}}$ Mohini Parmar, ${ }^{\mathrm{I}}$ Ernie Wong, ${ }^{\mathrm{H}}$ Loong-Yuan Han ${ }^{\mathrm{J}}$ and Sarah L Elkin ${ }^{\mathrm{K}}$
}

\begin{abstract}
Integration of primary and secondary care for the management of respiratory disease is a long-held ambition. Here, we describe how respiratory specialists at a large NHS trust, working with primary care clinicians in the area, set up a GP hotline and respiratory support service in response to the COVID-19 pandemic, with the aim of enhancing delivery of care to patients in this unprecedented time. Working across traditional organisational boundaries in this way confers benefits to patients and clinicians, illustrating the value of new, integrated models of care.
\end{abstract}

KEYWORDS: COVID-19, respiratory medicine, integrated care, primary care, telephone hotline

DOI: $10.7861 /$ fhj.2020-0082

Authors: Aspecialty trainee in respiratory medicine and general internal medicine, Imperial College Healthcare NHS Trust, London, UK and NIHR Imperial BRC clinical research fellow in respiratory medicine, National Heart and Lung Institute, Imperial College London, London, UK; ${ }^{B}$ Consultant physician in respiratory integrated care, Imperial College Healthcare NHS Trust, London, UK and clinical director of the London Respiratory Strategic Clinical Network; ' specialty trainee in respiratory medicine and general internal medicine, Imperial College Healthcare NHS Trust, London, UK; ${ }^{D}$ GP principal, Golborne Medical Centre, London, UK; Econsultant physician in respiratory medicine, Imperial College Healthcare NHS Trust, London, UK; F deputy director of transformation, Imperial College Healthcare NHS Trust, London, UK; ${ }^{6}$ integrated care programme director, Imperial College Healthcare NHS Trust, London, UK; ${ }^{H}$ Consultant physician in respiratory medicine, Imperial College Healthcare NHS Trust, London, UK and honorary clinical senior lecturer, National Heart and Lung Institute, Imperial College London, London, UK; ' $G$ P partner, Barnabas Medical Centre, London, UK and chair, NHS Ealing Clinical Commissioning Group; 'associate specialist in respiratory medicine, Imperial College Healthcare NHS Trust, London, UK; ${ }^{k}$ consultant physician in respiratory medicine and clinical director of integrated care, Imperial College Healthcare NHS Trust, London, UK, and honorary clinical senior lecturer, National Heart and Lung Institute, Imperial College London, London, UK

\section{Introduction}

Closer collaboration and joint working across primary, community and secondary healthcare sectors has been openly encouraged in the drive for new, patient-centred models of care. It has been suggested that hospital specialists should become part of a wider network of care with a key role in overseeing governance. Sustained and large-scale change at a system level has been challenging due to a number of barriers, including financially driven organisational boundaries and traditional ways of working. The COVID-19 pandemic has resulted in profound shifts in care delivery models and opportunities to work differently within a period of uncertainty.

In North West London, as elsewhere, it became rapidly apparent that COVID-19 was placing extraordinary demands on services and individuals in primary and secondary healthcare settings. Frontline clinicians have been facing an array of clinical, ethical and logistical challenges on a daily basis. Primary care clinicians were being required to manage suspected and confirmed cases of COVID-19 in patients presenting with an array of symptoms and varying degrees of haemodynamic stability or respiratory compromise, potentially on a background of multiple comorbidities. ${ }^{2}$ Outpatient departments were closed and usual pathways of care and advice no longer available.

Telephone hotlines have long been established and may improve communication between GPs and hospital specialists, ${ }^{3}$ which is vital for reducing diagnostic delays and providing effective continuity of care. ${ }^{4}$ Such services have been shown to be feasible and effective in various specialities. Evaluation of a paediatric hotline previously set up at our Trust showed that outpatient referrals were either better directed or could be avoided following discussion between GPs and paediatric specialists. ${ }^{5}$ An audit of a telephone hotline set up for transient ischaemic attacks and strokes showed that the service resulted in a marked reduction in delays in the time taken to clinical review and treatment. ${ }^{6}$ Similarly, a telephone hotline set up for rapid referrals for suspected metastatic cord compression resulted in a considerable reduction in the median time from referral to diagnosis.? However, uptake of such services may be variable, as demonstrated by a specialist orthopaedic telephone hotline for GPs that was infrequently utilised. ${ }^{8}$

Understanding of COVID-19 continues to evolve rapidly. The aim of this short report is to highlight the value of a telephone support 
hotline for COVID-19 and respiratory enquiries that is linked to regular educational webinars, and to encourage respiratory services across the UK to include this in their care delivery model moving forward in preparation for any future COVID-19 surges.

\section{Solution}

There was no pathway in place in North West London to provide GPs with respiratory specialist support in relation to COVID-19. Additionally, our institution has not offered a respiratory advice line service such as this before. Previously, requests for specialist respiratory advice from GPs would either have been sent in written or e-mail form directly to respiratory consultants or in more acute situations would have been directed to the on-call medical registrar.

To address a gap in support for primary care during the COVID-19 crisis, we set up a respiratory specialist advice telephone hotline service that is accessible to all GPs in the North West London health and care partnership, comprising $400 \mathrm{GP}$ practices serving a population of more than 2 million residents. It quickly became apparent that facilitated learning through webinars enabled sharing of lessons learnt and complemented the hotline service.

When GPs telephone the advice line, they are connected to the responsible respiratory clinician's mobile phone via an automated system that is part of our hospital switchboard. The advice line, open daily from 9 am to $6 \mathrm{pm}$, is staffed by a respiratory consultant and a respiratory specialty trainee (grade ST4 or above and deemed to have sufficient respiratory experience to provide advice) on weekdays, including bank holidays, and by a respiratory physician at weekends. To minimise disruption while undertaking patient-facing activities such as clinic or ward duties, the respiratory clinicians are only scheduled to cover the advice line service during their clinical administration hours. The respiratory specialty trainees can directly contact the supervising consultant at any time to discuss any queries for an immediate response.

The service has been advertised through the sustainability and transformation partnership (STP) and clinical commissioning group (CCG) communication bulletins, social media and word of mouth.
The aim of the initiative has been to provide specialist input, second opinions and practical advice on managing new and challenging clinical situations, both in relation to COVID-19 but also in the context of other general, non-COVID-19 respiratory queries. To ensure rapid dissemination of lessons learnt, we deliver a regular webinar for GPs in the sector to discuss the key themes emerging from calls to the service and have used this information to regularly update local guidelines on the management of COVID-19 in the community.

\section{Results}

The hotline launched on 6 April 2020. In the first 35 days of setting up the service, a total of 310 calls were received: 288 were answered; 20 were abandoned by the caller before the call could be answered; and two were redirected if no doctors were logged into the system at the time of the call (for example, if users called the service outside its opening hours). Out of the 288 answered calls, we responded to 197 unique queries from GPs across various clinical commissioning groups (CCGs) in the sector. The mean call duration was 5 minutes 43 seconds. There was a mean of 7.7 unique queries per weekday (range 4-12) and 0.4 queries per day on weekends (range $0-3$ ).

Several themes emerged from the telephone calls (Table 1 and Fig 1). The most frequent queries have related to the management of persisting COVID-19 symptoms lasting for several weeks including chest pain, cough, breathlessness, fever, palpitations and fatigue - and to initial investigations for abnormal observations (tachypnoea, hypoxia, tachycardia or fever, either in isolation or combination). Several calls have sought clarification on existing community services and pathways that are available to patients during the COVID-19 pandemic, including questions on accessing home oxygen services or palliative care support. Additional clarification has frequently been sought on current shielding and self-isolation guidelines. We have also provided advice on a range of general respiratory queries not relating to COVID-19. These have pertained particularly to management of asthma, chronic obstructive pulmonary disease and bronchiectasis. Second opinions regarding the appropriateness of referrals to secondary care, use of antibiotics and interpretation of abnormal chest radiology have also been sought.

Table 1. Frequency of calls to the advice line by week

\begin{tabular}{|c|c|c|c|c|c|c|}
\hline \multirow[t]{3}{*}{ Theme } & \multicolumn{6}{|c|}{ Number of calls received } \\
\hline & Days & Days & Days & Days & Days & Total \\
\hline & $1-7$ & $8-14$ & $15-21$ & $22-28$ & 29-35 & \\
\hline Persisting symptoms & 12 & 9 & 13 & 15 & 17 & 66 \\
\hline Initial investigations for abnormal observations & 8 & 9 & 11 & 11 & 2 & 41 \\
\hline General respiratory queries & 9 & 2 & 12 & 7 & 4 & 34 \\
\hline Community services and pathways & 5 & 4 & 2 & 2 & 2 & 15 \\
\hline $\begin{array}{l}\text { Appropriateness of referrals to secondary care for } \\
\text { acute medical assessment }\end{array}$ & 2 & 2 & 3 & 0 & 5 & 12 \\
\hline Shielding and self-isolation guidance & 0 & 2 & 4 & 2 & 1 & 9 \\
\hline Antibiotics & 1 & 1 & 3 & 3 & 0 & 8 \\
\hline Abnormal radiology & 3 & 1 & 1 & 1 & 2 & 8 \\
\hline Extra-pulmonary manifestations of COVID-19 & 0 & 2 & 1 & 0 & 0 & 3 \\
\hline Miscellaneous & 1 & 0 & 0 & 0 & 0 & 1 \\
\hline Total & 41 & 32 & 50 & 41 & 33 & 197 \\
\hline
\end{tabular}




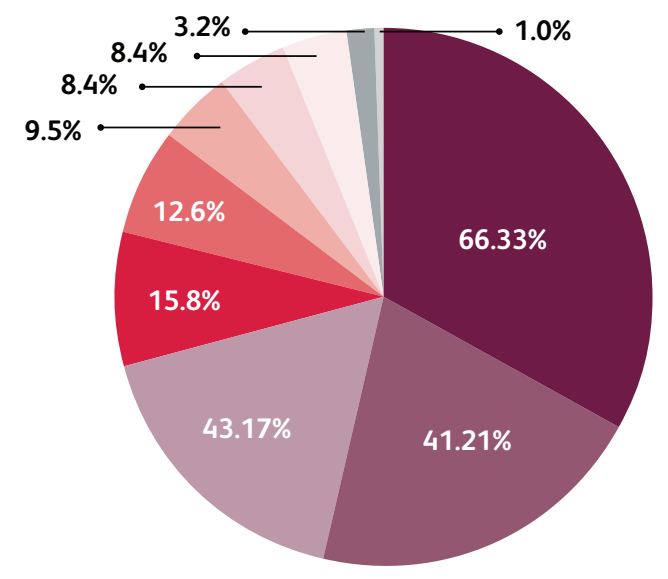

- Persisting symptoms

abnormal observations

General respiratory queries

- Community services and pathways

- Appropriateness of referrals to secondary care for acute medical assessment

Fig 1. Themes of calls to the advice line over the first 35 days.

The nature of the queries received changed over time. The number of calls seeking advice about further investigations for abnormal test results markedly decreased by the fifth week, whereas calls about management of persisting symptoms increased. The former finding likely reflects increased awareness and confidence among clinicians in managing initial symptoms of COVID-19 as the weeks have gone by, while the latter use of the service is likely indicative of the newly emerging challenge of managing medium-to-long-term complications of the infection.

\section{Evaluation}

Feedback from GPs who have used the service has been encouraging. Satisfaction with the service received a mean score of $9.3 / 10(n=41)$. GPs have felt that the hotline has been effective at changing clinical management, providing ease of access to specialist advice, improving confidence in managing respiratory manifestations of COVID-19, ensuring coordination of care, and helping GPs to feel supported (Fig 2). The service has been described by GPs as 'very helpful and supportive, especially if considering sending in a patient or as a sounding board about COVID-19' and has been appreciated for its 'accessibility to specialist discussion' and 'quick senior clinician response'. Each of the three webinars conducted thus far, in which key lessons have been disseminated to GPs, have been attended by between 120180 clinicians. They have achieved mean scores of 9.4/10 for being informative, 9.4/10 for being useful and 9.0/10 for being convenient $(n=51)$. There is appetite among GPs for the webinars to continue during the recovery from COVID-19 to facilitate learning together. Owing to low service uptake at weekends, the weekend service has now been stopped. The telephone calls received are not arduous and will continue to be incorporated into clinicians' administration time. There is a desire among the respiratory consultants involved in the service to keep the hotline operational.

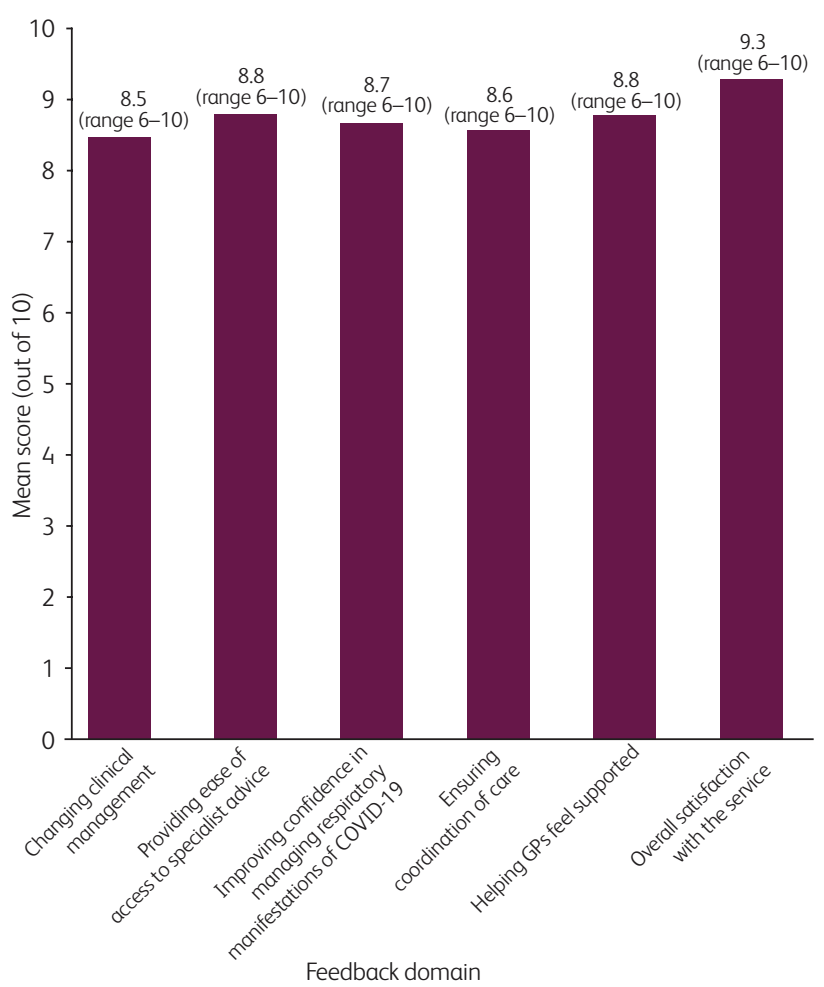

Fig 2. GP feedback scores on the effectiveness of the advice line.

Our telephone hotline offers a simple way in which specialists can provide direct support to primary care services in the challenging COVID-19 climate. There are multiple benefits to setting up such a specialist telephone hotline (Box 1).

\section{Box 1. Benefits and challenges in setting up a respiratory specialist hotline for primary care}

\section{Benefits}

> Forges new connections with primary care and strengthens relationships, thus expanding clinical networks

> Supports primary care clinicians with management plans for respiratory patients in the community

$>$ Enables timely, joined up and appropriate care to be delivered to the patient, leading to a better patient experience

$>$ Negates the need for a respiratory outpatient appointment or bridges the gap until the next respiratory review

> Enhances flow of patients through appropriate care pathways in the community and in secondary care

> Offers a novel way for respiratory trainees to develop their clinical decision-making, communication and specialist medicine skills

\section{Challenges}

> Availability of respiratory specialists to staff the hotline (job planning)

> Need to streamline information flow and appropriately manage time if service usage is high

$>$ Ensuring appropriate governance of the system, risk reporting and record keeping

> Uncertainties when providing advice related to COVID-19 due to the rapidly evolving evidence 


\section{Benefits to patients and clinicians}

By offering a specialist opinion regarding clinical management, the advice line provides colleagues in primary care with reassurance about their planned treatment and interventions. Some GPs reported that if they did not have access to the service, they would have 'felt very under-confident' and 'felt more anxious' about the clinical decision-making, and they would have either asked GP colleagues for advice or attempted to contact their local on-call medical registrar, respiratory registrar or ambulatory care clinic. The advice line helps to ensure that patients are directed towards the most appropriate care pathways by reducing emergency department attendance of those patients who could continue to be managed in the community, while also ensuring that those who do warrant urgent medical assessment are directed to secondary care emergency services when appropriate. Given the reduction in specialist outpatient services during the COVID-19 outbreak, the advice line also bridges the gap until the next outpatient appointment by providing interim advice on ongoing management of various respiratory conditions. In addition to receiving calls from GPs, we have received queries directly from GP practice nurses and community pharmacists, suggesting that members of the wider multidisciplinary team have felt a need for respiratory specialist advice. This has offered an opportunity to forge new connections with GP practices and multidisciplinary team members, while also strengthening longestablished relationships with primary care, thus expanding and reinforcing our clinical network.

\section{Benefits to trainees}

From a respiratory trainee perspective, staffing the advice line is an invaluable educational experience, drawing upon a range of medical and communication skills, while promoting development of trainee autonomy through a novel form of clinical triaging and decision-making. It provides considerable insight into the heterogeneity and multisystemic nature of COVID-19 presentations, as well as an opportunity to reinforce understanding of management steps for common respiratory conditions unrelated to COVID-19, thus linking directly to both respiratory and general internal medicine competencies. It has also provided exposure to an array of thought-provoking clinical scenarios and offered an innovative means for continued learning and development during the COVID-19 outbreak, during which time certain traditional training opportunities have naturally been curtailed.

The COVID-19-related questions to the advice line have required clinical decision-making that would normally fall under the remit of the physicians on the medical team rather than the wider multidisciplinary team. For this reason, the service has been staffed by physicians. However, we have suggested referral onwards to other members of the multidisciplinary team where clinically appropriate, thus encouraging engagement with a range of healthcare professionals and potentially creating learning opportunities for other such professionals in training.

\section{Potential challenges}

Logistical challenges to setting up such a service include securing time in job plans and ensuring provision of respiratory specialists with the necessary skill sets. It is important to streamline information flow and appropriately manage time if service usage is high. We have found that it is entirely feasible to cover the hotline service during clinical administration hours as the number of calls received per day is not overly burdensome. The advice line service does not require a standalone clinical session for the covering clinicians. Additionally, there needs to be a system for governance, risk reporting and record keeping, which may be challenging if joint records systems between primary and secondary care are unavailable. Funding models that are currently challenging will benefit from future plans for crossorganisational integrated care systems.

We acknowledge that understanding of COVID-19 is rapidly evolving and there may be a degree of uncertainty in the evidence base for the advice that is being given. However, the priority has always been to provide safe, well-reasoned care that maintains patient safety at its core and to learn together. Current uncertainties regarding the evidence were actively discussed and acknowledged when the advice was offered.

\section{The quadruple aim}

As highlighted by Berwick and colleagues, provision of highquality integrated care requires health systems to strive for the 'triple aim' of improving patients' experience of care, improving population health and lowering per capita healthcare costs. ${ }^{9}$ More recently, this notion has evolved into the 'quadruple aim', which acknowledges the additional goal of improving the experience of healthcare providers themselves. ${ }^{10}$ We have strived to achieve these aims by engaging respiratory physicians in a novel way of working and thereby easing some of the burden placed on clinicians in primary care. ${ }^{11}$ The advice line was not specifically designed to assess secondary care physician experience or set up with the aim of assessing cost effectiveness. It was a rapid response to the COVID-19 crisis rather than a project to release funds. Nevertheless, our advice line has sought to align to the principles of the quadruple aim by promoting:

> positive patient experience - improved by knowledge that specialists are involved in the decision being made ${ }^{12,13}$

> improved clinician experience - by growth of inter-professional relationships and respect

> better value - by providing increased accessibility to specialist advice, subsequently leading (where clinically appropriate) to decreased need for hospital attendance

> improved patient outcomes - by helping to ensure that patients receive appropriate and timely care that adhere to current guidelines. ${ }^{14}$

\section{The future}

Healthcare systems should aim to provide coordinated, integrated care for patients, both during the COVID-19 crisis and beyond. Integration of primary and secondary care for the management of respiratory disease, especially long-term condition management, is particularly desirable. ${ }^{15}$ The COVID-19 pandemic may provide the impetus to achieve this.

Our innovation appears to demonstrate that by linking a telephone hotline service to educational webinars and by addressing common themes, it is possible to ensure ongoing learning and potentially promote to a sustained improvement in condition management within the primary care space. As our understanding of COVID-19 improves, the frequency of COVID19-related queries to the advice line is decreasing. However, as suggested by our data so far, there is still value in such a service 
continuing in order to support any future COVID-19 surges and to provide general respiratory advice unrelated to COVID-19. This model can also be expanded to other medical specialties.

Our intervention is one of many ways to help deliver robust integrated care with specialists working across institutional boundaries. ${ }^{16}$ We strongly encourage the development of a similar model of care in other regions and inclusion of specialty trainees and other multi-professional learners in its design and delivery. By enhancing collaboration and improving both current and future relationships, it is hoped that safer and more effective care can be provided for patients in this unprecedented time, alongside an improvement in long-term condition management and stronger respiratory networks.

\section{Funding and conflicts of interest}

KK is supported by the NIHR Imperial Biomedical Research Centre (BRC). The views expressed are those of the author(s) and not necessarily those of the NIHR or the Department of Health and Social Care. EW has received travel grants from Chiesi and has spoken for GlaxoSmithKline. SLE has spoken in educational meetings for $\mathrm{AZ}$ and Chiesi.

\section{References}

1 Edwards N. The report of the Future Hospital Commission: first steps down the road to change? Future Healthc J 2014:1:13-15.

2 NHS London Clinical Networks. Primary care and community respiratory resource pack for use during COVID-19. NHS London Clinical Networks, 2020. www.pcrs-uk.org/sites/pcrs-uk.org/files/ resources/COVID19/Primary-and-Community-Care-RespiratoryResource-Pack-during-COVID-19-V5.pdf [Accessed 21 May 2020]

3 Harrison R, Clayton W, Wallace P. Can telemedicine be used to improve communication between primary and secondary care? BMJ 1996;313:1377-80.

4 Epstein RM. Communication between primary care physicians and consultants. Arch Fam Med 1995;4:403-9.

5 Watson M, Klaber R, Haynes C, Holt K, Lenman M. Paediatric consultant GP-hotline audit. Br J Gen Pract 2013;63:348.
6 Kerr E, Arulraj N, Scott M et al. A telephone hotline for transient ischaemic attack and stroke: prospective audit of a model to improve rapid access to specialist stroke care. BMJ 2010;341:c3265.

7 Allan L, Baker L, Dewar ] et al. Suspected malignant cord compression - improving time to diagnosis via a 'hotline': a prospective audit. Br J Cancer 2009:100:1867-72.

8 Roland M, Bewley B. Boneline: evaluation of an initiative to improve communication between specialists and general practitioners. J Public Health Med 1992;14:307-9.

9 Berwick DM, Nolan TW, Whittington J. The triple aim: care, health and cost. Health Aff (Millwood) 2008;27:759-69.

10 Sikka R, Morath JM, Leape L. The quadruple aim: care, health, cost and meaning in work. BMJ Qual Saf 2015;24:608-10.

11 Duffy B. 5 best practices to achieve the 'quadruple aim' and prevent physician burnout in the post-EHR era. Becker's Healthcare 2017. Available from: www.beckershospitalreview.com/healthcareinformation-technology/5-best-practices-to-achieve-the-quadrupleaim-and-prevent-physician-burnout-in-the-post-ehr-era.html [Accessed 21 May 2020].

12 NHS Institute for Innovation and Improvement. The Patient Experience Book. A collection of the NHS Institute for Innovation and Improvement's guidance and support. www.england.nhs.uk/ improvement-hub/wp-content/uploads/sites/44/2017/11/PatientExperience-Guidance-and-Support.pdf [Accessed 28 June 2020].

13 Waibel S, Vargas I, Coderch ], Vázquez M-L. Relational continuity with primary and secondary care doctors: a qualitative study of perceptions of users of the Catalan National Health System. BMC Health Serv Res 2018;18:257.

14 Woolf SH, Grol R, Hutchinson A, Eccles M, Grimshaw ]. Clinical guidelines: potential benefits, limitations, and harms of clinical guidelines. BMJ 1999;318:527-30.

15 Holmes WF, Macfarlane J. Issues at the interface between primary and secondary care in the management of common respiratory disease. Introduction. Thorax 1999;54:538-9.

16 Coughlan C, Manek N, Razak Y, Klaber RE. How to improve care across boundaries. BMJ 2020;369:m1045.

Address for correspondence: Dr Sarah L Elkin, Department of Respiratory Medicine, St Mary's Hospital, Imperial College Healthcare NHS Trust, Praed Street, London W2 1NY, UK. Email: Sarah.Elkin@nhs.net 